\title{
ESTABILIZACIÓN DE UNA BOLA SOBRE UN PLANO UTILIZANDO UN ROBOT PARALELO 6-RSS
}

\author{
Daniel González Lluís Ros Federico Thomas \\ Institut de Robòtica i Informàtica Industrial, UPC-CSIC \\ Llorens Artigas 4-6, 08028 Barcelona, Spain
}

\begin{abstract}
Resumen
Una bola rodando sobre un plano constituye un sistema no lineal cuya estabilización suele utilizarse en el campo de la ingeniería de control con propósitos de investigación y educativos. En las implementaciones habituales, la inclinación del plano se controla con dos motores, y la posición de la bola se detecta con una cámara o un sensor táctil que cubre el plano. Sin embargo, una actuación basada en un robot paralelo con 6 grados de libertad aumenta notablemente las posibilidades del sistema. En primer lugar, un robot como el mencionado permite rotar el plano con respecto a un eje instantáneo arbitrario del espacio; $y$, en segundo lugar, la utilización de sensores de fuerza en las seis cadenas serie del robot permite localizar la posición de la bola con precisión, sin tener que recurrir a sensores táctiles o cámaras externas. Basándonos en una formulación de la cinetostática del problema que utiliza la teoría de torsores, en este artículo mostramos cómo es posible derivar la relación entre las fuerzas y las velocidades en la plataforma y en los sensores y en los actuadores de forma simple y directa. A partir de esta formulación, se deriva un control basado en dos PIDs cuya efectividad es evaluada experimentalmente.
\end{abstract}

Palabras clave: Sistema bola sobre plano, teoría de torsores, robots paralelos.

\section{INTRODUCCIÓN}

Los sistemas dinámicos basados en una bola rodante sobre una guía $[10,15]$ y su extensión a la bola rodante sobre un plano $[7,11,12,16]$ son fáciles de implementar y dan a los estudiantes e investigadores la oportunidad de ensayar diferentes estrategias de control. En ambos casos el desafío consiste en estabilizar la bola ya sea en un punto de la guía o del plano, lo cual implica que la posición de la bola sea conocida en todo momento por medio de sensores, para así modificar la posición y orientación de la guía o del plano por medio de actuadores $[1,9,13]$.

Se han estudiado muchas estrategias de control

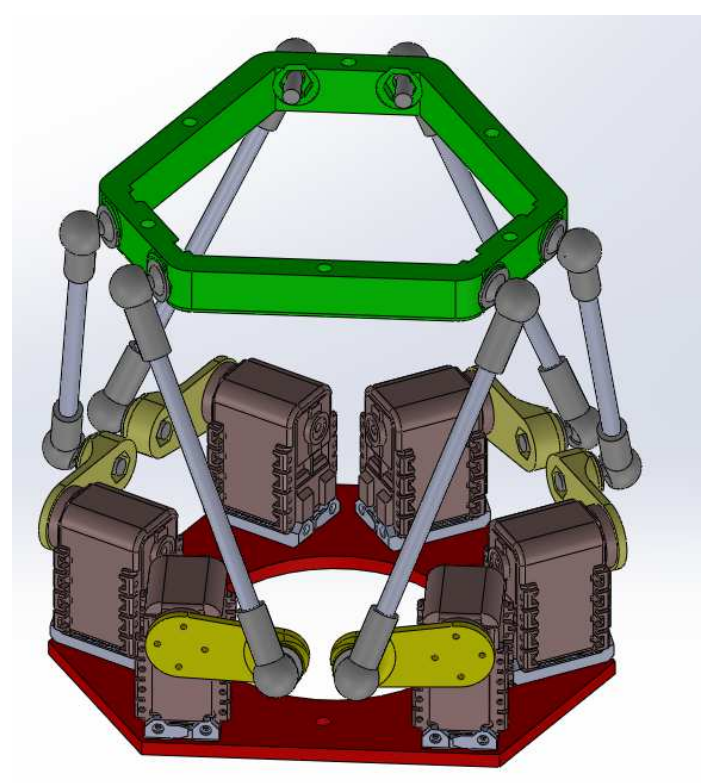

Figura 1: Diseño de un robot 6-․SS.

para este tipo de plantas, que incluyen, por ejemplo, el control de perturbaciones [16], el control difuso [7], el control por deslizamiento [12], o el control mediante redes neuronales [7].

En este trabajo no ahondaremos en el problemas del control sino en el diseño e implementación de la parte de actuación y sensado de la posición de la bola utilizando un robot paralelo. La utilización de una estructura paralela para el sensado del torsor de fuerzas aplicadas sobre una plataforma no es nueva [8] y puede ser utilizada, en el caso particular del problema tratado, para la localización de una bola sobre un plano.

Nos hemos decantado por la utilización de un robot paralelo con topología 6 - $\underline{R} S S$, una variante de la bien conocida plataforma de Gough-Stewart, por la simplicidad con que puede ser implementado utilizando motores de tipo servo. Este tipo de robots consiste en seis cadenas serie compuestas de una articulación de rotación actuada y anclada sobre una base fija y dos articulaciones esféricas, la última de las cuales está conectada a la plataforma móvil (véase la Fig. 1). Las seis articulaciones de 
rotación actuadas de forma independiente proveen a la plataforma móvil de seis grados de libertad, haciendo posible que ésta ejecute cualquier torsor instantáneo de velocidad dentro de su espacio de trabajo.

Este trabajo se ha organizado de la siguiente manera. En primer lugar, en la Sección 2 se analiza la cinetostática del robot paralelo. Utilizando la teoría de torsores, derivaremos las relaciones entre el torsor de velocidad en la plataforma y la velocidad angular de los motores, así como la relación entre el torsor de fuerza aplicado sobre la plataforma y la fuerza leída por los sensores. Esta formulación proporcionará el modelo utilizado para el control del sistema, tal como se describe en la Sección 3. A continuación, en la Sección 4 se describe la implementación del sistema hasta el punto en el que se encuentra en estos momentos y presenta algunos resultados experimentales ya obtenidos. Finalmente, la Sección 5 resume los puntos más importantes de este trabajo.

\section{ANÁLISIS CINETOSTÁTICO}

Para controlar el movimiento del robot se necesita el Jacobiano cinemático, que proporciona la relación entre la velocidad de la plataforma y las velocidades de los motores. Asimismo, para deducir la posición de la bola se requiere el Jacobiano estático, que proporciona el torsor de fuerza ejercido por la bola sobre la plataforma, dados los pares motor que lo equilibran. Este torsor tiene tres componentes de fuerza y tres de par y por tanto permite recuperar la recta de acción de la fuerza, y su punto de intersección con la plataforma. Obtendremos este punto y ambos Jacobianos constructivamente, apoyándonos en la teoría de torsores y el principio de las potencias virtuales $[5,6]$.

\subsection{ANÁLISIS DE LA VELOCIDAD}

Sea $\widehat{\boldsymbol{T}}=\left[\boldsymbol{\omega}^{\top}, \boldsymbol{v}_{O}^{\top}\right]^{\top}$ el torsor de velocidad absoluta de la plataforma, expresado en un sistema de referencia absoluto $\mathcal{A}=\{O X Y Z\}$ anclado a la base (Fig. 2). En la expresión de $\widehat{\boldsymbol{T}}, \boldsymbol{\omega} \in \mathbb{R}^{3}$ es la velocidad angular de la plataforma, y $\boldsymbol{v}_{O} \in \mathbb{R}^{3}$ es la velocidad del punto de la plataforma que coincide instantánemente con el origen $O$. Es bien sabido que este torsor se puede expresar como combinación lineal de los torsores articulares que aparecen a lo largo de cada pata del robot $[3,5,6]$. Consideremos por ejemplo la pata $i$, esquematizada en la Fig. 2. Cada una de sus articulaciones esféricas es equivalente a tres articulaciones rotacionales de ejes copuntuales, con lo cual la pata se puede ver como un brazo serie formado por siete enlaces, que podemos numerar consecutivamente desde la base (índice 0) hasta la plataforma (índice 7). Con esta convención, y utilizando la notación de Duffy [6], para la pata $i$ podemos escribir

$$
\widehat{\boldsymbol{T}}=\omega_{i, 1} \widehat{\boldsymbol{S}}_{i, 1}+\omega_{i, 2} \widehat{\boldsymbol{S}}_{i, 2}+\ldots+\omega_{i, 7} \widehat{\boldsymbol{S}}_{i, 7},
$$

donde $\omega_{i, j}$ y $\widehat{\boldsymbol{S}}_{i, j}$ son la velocidad angular y el torsor de velocidad unitario del enlace $j+1$ relativos al enlace $j$ de la pata. Nótese que en esta expresión $\omega_{i, 1}$ es la velocidad angular del motor $i, \mathrm{y}$ $\widehat{\boldsymbol{S}}_{i, 1}$ se puede ver como el vector de coordenadas de Plücker normalizadas del eje de este motor.

Escribiendo la Eq. (1) para las seis patas se obtiene un sistema de 36 ecuaciones lineales en 42 incógnitas que en principio ya se podría usar para resolver el problema cinemático inverso instantáneo (dado $\widehat{\boldsymbol{T}}$, obtener $\omega_{i, 1}$ para $\left.i=1, \ldots, 6\right)$. Este sistema, sin embargo, está subdeterminado, y en realidad se puede simplificar mucho como vamos a ver, reduciendo así el tiempo de cálculo de la ley de control resultante. La simplificación se efectúa mediante el método del torsor recíproco $[3,6]$.

Sea $\widehat{\boldsymbol{w}}_{i}$ el torsor unitario formado por las coordenadas de Plücker de la recta que pasa por los puntos $B_{i}$ y $C_{i}$ de la pata $i$. Este torsor tiene la forma

$$
\widehat{\boldsymbol{w}}_{i}=\left[\begin{array}{c}
\boldsymbol{e}_{i} \\
\boldsymbol{r}_{i} \times \boldsymbol{e}_{\boldsymbol{i}}
\end{array}\right]
$$

donde $\boldsymbol{e}_{i}$ es un vector director unitario de la recta $B_{i} C_{i}$, y $\boldsymbol{r}_{i}$ es el vector de posición del punto $B_{i}$. Si ahora multiplicamos la Eq. (1) por $\widehat{\boldsymbol{w}}_{i}^{\top}$, obtendremos

$$
\widehat{\boldsymbol{w}}_{i}^{\top} \cdot \widehat{\boldsymbol{T}}=\omega_{i, 1} \cdot \widehat{\boldsymbol{w}}_{i}^{\top} \widehat{\boldsymbol{S}}_{i, 1}
$$

puesto que todos los productos $\widehat{\boldsymbol{w}}_{i}^{\top} \widehat{\boldsymbol{S}}_{i, j}$ son nulos para $j=2, \ldots, 7$. Esto es así porque la recta asociada a $\widehat{\boldsymbol{w}}_{i}$ intersecta las rectas de los torsores asociados a las rótulas esféricas. Debido a esta propiedad, se dice que $\widehat{\boldsymbol{w}}_{i}$ es el torsor recíproco de la pata $i$.

Si ahora escribimos la Eq. (3) para todas las patas, obtendremos el siguiente sistema de ecuaciones

$$
\begin{aligned}
\underbrace{\left[\begin{array}{ccc}
\boldsymbol{e}_{1} & \ldots & \boldsymbol{e}_{6} \\
\boldsymbol{r}_{1} \times e_{1} & \ldots & \boldsymbol{r}_{6} \times e_{6}
\end{array}\right]^{\top}}_{\mathbf{A}} \cdot \underbrace{\left[\begin{array}{c}
\boldsymbol{\omega} \\
\boldsymbol{v}_{O}
\end{array}\right]}_{\widehat{\boldsymbol{T}}}= \\
\underbrace{\left[\begin{array}{ccc}
\widehat{\boldsymbol{w}}_{1}^{\top} \cdot \widehat{\boldsymbol{S}}_{1,1} & \\
& \ddots & \\
& & \widehat{\boldsymbol{w}}_{6}^{\top} \cdot \widehat{\boldsymbol{S}}_{6,1}
\end{array}\right]}_{\mathbf{B}} \cdot \underbrace{\left[\begin{array}{c}
\omega_{1,1} \\
\vdots \\
\omega_{6,1}
\end{array}\right]}_{\boldsymbol{\gamma}}
\end{aligned}
$$

que podemos expresar de forma compacta como

$$
\mathbf{A} \widehat{T}=\mathbf{B} \gamma
$$

expresión que proporciona, directamente, la relación entre las velocidades de entrada y salida del 


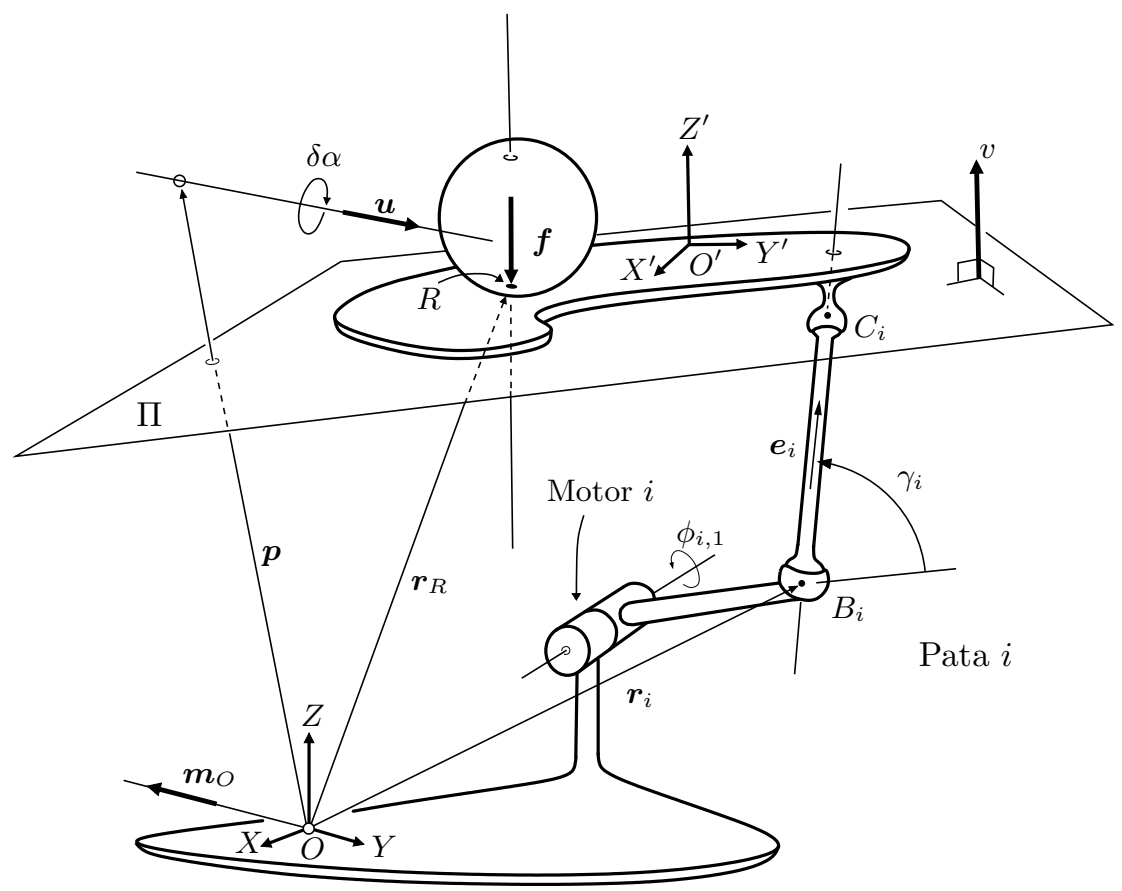

Figura 2: Elementos geométricos que intervienen en el análisis cinetostático del robot.

robot. Si ahora suponemos que $\operatorname{det} \mathbf{B} \neq 0$, podemos finalmente escribir la solución del problema cinemático instantáneo inverso como

$$
\gamma=\mathbf{B}^{-1} \mathbf{A} \widehat{T}
$$

Obsérvese que la matriz $\mathbf{B}$ es diagonal, y sólo puede ser singular si alguno de los productos $\widehat{\boldsymbol{w}}_{j}^{\mathrm{T}} \cdot \widehat{\boldsymbol{T}}_{j, 1}$ se hace cero, lo cual sólo sucede cuando la recta $B_{i} C_{i}$ intersecta el eje del motor $i$. En tal situación, se dice que el robot se encuentra en una singularidad inversa, produciéndose una pérdida de destreza de la plataforma móvil [2-4]. Si bien esta situación podría darse en la práctica, en este trabajo evitaremos que ocurra limitando los ángulos $\gamma_{i}$ entre el brazo del motor y la recta $B_{i} C_{i}$ a un subrango del intervalo $(0, \pi / 2)$.

Fijémonos ahora que la Eq. (6) se puede multiplicar por un incremento infinitesimal de tiempo $\delta t$, dando lugar a la ecuación

$$
\delta \phi=\mathbf{B}^{-1} \mathbf{A} \delta \widehat{D} .
$$

que proporciona los pequeños desplazamientos $\delta \phi$ que deben realizar los motores para generar un desplazamiento infinitesimal $\delta \widehat{\boldsymbol{D}}$ de la plataforma. Esta es, precisamente, la ecuación a implementar en nuestro lazo de control.

Por un lado, el vector $\delta \phi$ tiene la forma

$$
\delta \phi=\left[\delta \phi_{1}, \ldots, \delta \phi_{6}\right],
$$

siendo $\delta \phi_{i}$ el pequeño ángulo que debe girar el motor $i$. Por otro lado, en virtud del Teorema de
Chasles, el torsor $\delta \widehat{\boldsymbol{D}}$ se puede descomponer como una rotación de ángulo infinitesimal $\delta \alpha$ en torno al eje instantáneo de rotación y deslizamiento de la plataforma, más una traslación infinitesimal de valor $\delta d$ a lo largo de este eje. Sean $\boldsymbol{u}$ un vector director unitario de este eje instantáneo, y $\boldsymbol{p}$ el vector de posición de un punto cualquiera del eje. Entonces, la descomposición mencionada se puede escribir así

$$
\delta \widehat{\boldsymbol{D}}=\delta \alpha \cdot \delta \widehat{\boldsymbol{D}}_{r}+\delta d \cdot \delta \widehat{\boldsymbol{D}}_{t}
$$

donde

$$
\delta \widehat{\boldsymbol{D}}_{r}=\left[\begin{array}{c}
\boldsymbol{p} \times \boldsymbol{u} \\
\boldsymbol{u}
\end{array}\right], \quad \delta \widehat{\boldsymbol{D}}_{t}=\left[\begin{array}{l}
\boldsymbol{u} \\
\mathbf{0}
\end{array}\right]
$$

En resumen, en cada iteración del lazo de control haremos lo siguiente:

1. A partir del error de posición de la bola, fijaremos el eje instantáneo alrededor del cual debe rotar la plataforma para corregir este error, y estableceremos los incrementos correctores $\delta \alpha$ y $\delta d$ necesarios.

2. Obtendremos $\delta \widehat{\boldsymbol{D}}$ usando la Eq. (9).

3. Determinaremos $\delta \boldsymbol{\phi}=\left[\phi_{1}, \ldots, \phi_{6}\right]^{\top}$ mediante la Eq. (7).

4. Consignaremos los incrementos $\delta \phi_{i}$ obtenidos en cada uno de los seis motores. 


\subsection{ANÁLISIS DE LA FUERZA}

Sea $\widehat{\boldsymbol{w}}$ el torsor de fuerza que aplica la bola sobre la plataforma móvil. Puesto que el contacto de la bola con la plataforma es puntual, podemos suponer que $\widehat{\boldsymbol{w}}$ codifica una fuerza pura $\boldsymbol{f}$ aplicada en el punto de contacto $R$, de momento $\boldsymbol{m}_{O}$ con respecto a $O$ (Fig. 2). Es decir, $\widehat{\boldsymbol{w}}=\left[\boldsymbol{f}^{\top}, \boldsymbol{m}_{O}^{\top}\right]^{\top}$. Veamos ahora como podemos deducir $\widehat{\boldsymbol{w}}$ a partir de los pares equilibrantes ejercidos por los motores, y como se puede obtener la posición de $R$ sobre el plano $\pi$ de la plataforma a partir de los vectores $\boldsymbol{f}$ y $\boldsymbol{m}_{O}$.

Supongamos que en un cierto instante la bola está aplicando el torsor $\widehat{\boldsymbol{w}}$ sobre la plataforma, la cual, a su vez, se mueve bajo una cierta velocidad $\widehat{\boldsymbol{T}}$. En ese instante, cada motor $i$ del robot aplicará un par equilibrante $\tau_{i}$ para equilibrar $\widehat{\boldsymbol{w}}$. Si asumimos que las patas y la plataforma tienen masa despreciable en comparación con la de la bola, es fácil aplicar el teorema de las potencias virtuales para deducir los Jacobianos de fuerza. A tenor del teorema, la potencia generada por $\widehat{\boldsymbol{w}}$ bajo la velocidad $\widehat{\boldsymbol{T}}$ debe ser igual a la potencia generada por los pares $\tau_{i}$ bajo las velocidades $\omega_{i}$ (se supone que las restricciones de enlace son ideales y no generan, en conjunto, potencia virtual neta). Es decir,

$$
\widehat{\boldsymbol{w}}^{\top} \cdot \widehat{\boldsymbol{T}}=\boldsymbol{\tau}^{\top} \cdot \gamma,
$$

donde $\boldsymbol{\tau}=\left[\tau_{1}, \ldots, \tau_{6}\right]$. Pasando el segundo miembro al lado izquierdo, esta ecuación se puede escribir de la siguiente manera

$$
\left[\widehat{\boldsymbol{w}}^{\top},-\boldsymbol{\tau}^{\top}\right] \cdot\left[\begin{array}{l}
\widehat{\boldsymbol{T}} \\
\gamma
\end{array}\right]=\mathbf{0}
$$

La Eq. (6) también se puede escribir de manera parecida

$$
[\mathbf{A},-\mathbf{B}] \cdot\left[\begin{array}{l}
\widehat{T} \\
\gamma
\end{array}\right]=\mathbf{0}
$$

y comparando estas dos últimas ecuaciones nos damos cuenta de que el vector $\left[\widehat{\boldsymbol{w}}^{\top},-\boldsymbol{\tau}^{\top}\right]$ debe ser, necesariamente, una combinación lineal de las filas de $[\mathbf{A},-\mathbf{B}]$. En otras palabras, $\widehat{\boldsymbol{w}}$ y $\boldsymbol{\tau}$ serán compatibles si y sólo si existe un vector $\boldsymbol{\beta} \in \mathbb{R}^{6}$ tal que

$$
\left[\begin{array}{c}
\widehat{\boldsymbol{w}} \\
-\boldsymbol{\tau}
\end{array}\right]=\left[\begin{array}{c}
\mathbf{A}^{\top} \\
-\mathbf{B}^{\top}
\end{array}\right] \boldsymbol{\beta},
$$

o, equivalentemente, si y sólo si

$$
\begin{aligned}
\widehat{\boldsymbol{w}} & =\mathbf{A}^{\top} \boldsymbol{\beta}, \\
\boldsymbol{\tau} & =\mathbf{B}^{\top} \boldsymbol{\beta} .
\end{aligned}
$$

Dado que en nuestro caso $\operatorname{det} \mathbf{B} \neq 0$, podemos aislar el vector $\boldsymbol{\beta}$ de la Eq. (16) y substituirlo en la Eq. (15), llegando a

$$
\widehat{\boldsymbol{w}}=\mathbf{A}^{\top} \mathbf{B}^{-1} \boldsymbol{\tau}
$$

que proporciona la relación buscada para $\widehat{\boldsymbol{w}}$.

Una vez hemos obtenido $\widehat{\boldsymbol{w}}$, es fácil recuperar las coordenadas del punto $R$ de contacto de la bola con la plataforma. Fijémonos que este punto se encuentra en la intersección de la recta de acción del torsor $\widehat{\boldsymbol{w}}$ con el plano $\Pi$ de la plataforma. $\mathrm{Al}$ ser $\widehat{\boldsymbol{w}}$ un torsor de fuerza pura, sus componentes $\left[\boldsymbol{f}^{\top}, \boldsymbol{m}_{O}^{\top}\right]^{\top}$ dan directamente las coordenadas de Plücker de la recta soporte. Si suponemos que la ecuación de $\Pi$ viene dada por $v_{0}+\boldsymbol{v} \cdot \boldsymbol{x}=0$, donde $\boldsymbol{v} \in \mathbb{R}^{3}$ es un vector unitario normal al plano, y $v_{0} \in \mathbb{R}$ es la distancia mínima de $O$ a $\Pi$, el vector de posición de $R$ viene dado por

$$
\boldsymbol{r}_{R}=\frac{\boldsymbol{v} \times \boldsymbol{m}_{O}-v_{0} \cdot \boldsymbol{f}}{\boldsymbol{v} \cdot \boldsymbol{f}}
$$

que es una expresión bien conocida en el ámbito de la geometría computacional de rectas [14, pág. 138]:

\section{SISTEMA DE CONTROL}

Una vez halladas las ecuaciones que nos permiten conocer la posición de la bola y la relación entre los incrementos de posición en la plataforma y los ángulos de los motores, el siguiente paso es proponer un sistema de control que nos permita estabilizar la bola en el baricentro de la plataforma móvil.

El sistema de control propuesto se esquematiza en la Fig. 3 y tiene dos partes bien diferenciadas: el sistema de sensado del error, que permite obtener el error de posición de la bola, y el sistema de corrección de la posición, que genera las señales de control necesarias para reducir este error. Veamos cada uno de estos dos sistemas.

\subsection{SENSADO DEL ERROR}

Para medir el error de posición de la bola, definimos un sistema de referencia $\mathcal{P}=\left\{O^{\prime} X^{\prime} Y^{\prime} Z^{\prime}\right\}$ anclado a la plataforma móvil, con origen $O^{\prime}$ en su baricentro, y ejes $O^{\prime} X^{\prime}$ y $O^{\prime} Y^{\prime}$ paralelos al plano $\Pi$ (Fig. 2). Utilizando este sistema, nuestro problema de control se reduce a estabilizar la bola en el origen $O^{\prime}$ de $\mathcal{P}$. El error de posición, por tanto, vendrá dado por el vector de posición de $R$ en $\mathcal{P}$, $r_{R}^{\mathcal{P}}$. Es fácil ver que

$$
\boldsymbol{r}_{R}^{\mathcal{P}}=\mathbf{R}^{\top} \cdot\left(\boldsymbol{r}_{R}-\boldsymbol{r}_{O^{\prime}}\right),
$$

donde $\boldsymbol{r}_{O^{\prime}}$ es la posición absoluta de $O^{\prime}$, y $\mathbf{R}$ es la matriz de rotación que proporciona la orientación absoluta de $\mathcal{P}$.

Sean $e_{x}$ y $e_{y}$ las componentes $X^{\prime}$ y $Y^{\prime}$ de $\boldsymbol{r}_{R}^{\mathcal{P}}$. Aunque podríamos tomar la cantidad $\sqrt{e_{x}^{2}+e_{y}^{2}}$ 


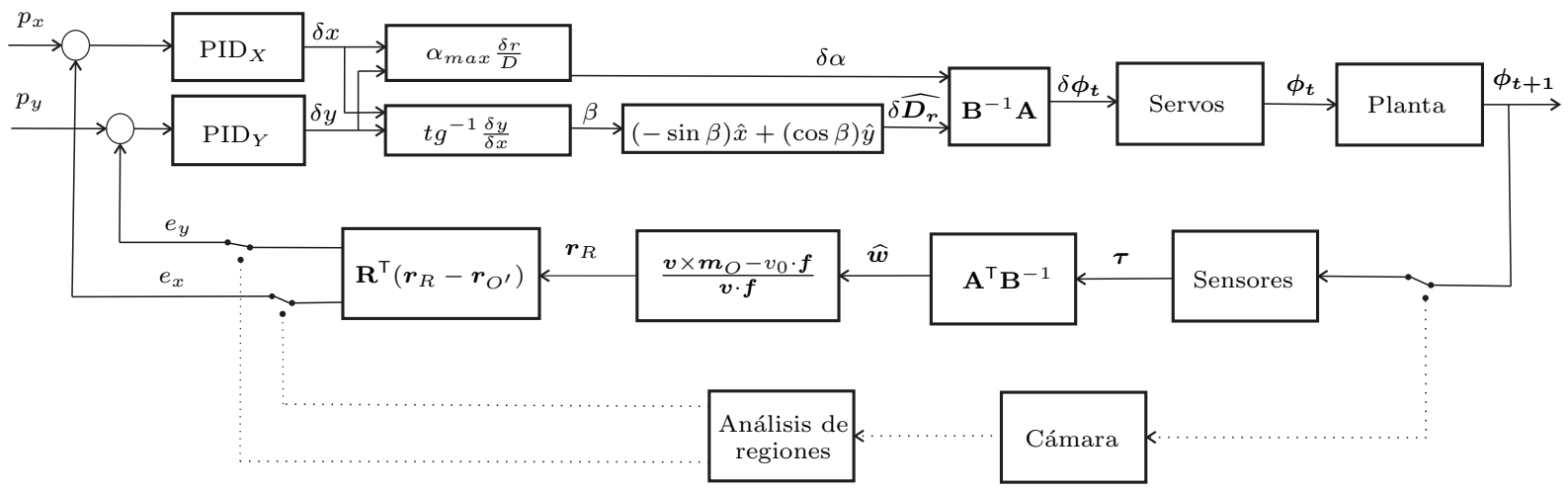

Figura 3: Sistema de control

como medida global del error, preferimos utilizar dos controladores PID independientes que reduzcan, respectivamente, los errores $e_{x} \mathrm{y} e_{y}$. Es decir controlamos las dos cordenadas de la posición de la bola en el plano del mismo modo que se hace, por ejemplo, en [13].

La rama intermedia de la Fig. 3 esquematiza, de derecha a izquierda, el cálculo de $e_{x}$ y $e_{y}$, que consta de los pasos siguientes:

1. Los sensores de los motores proporcionan los pares $\boldsymbol{\tau}=\left[\tau_{1}, \ldots, \tau_{6}\right]$.

2. A partir de $\boldsymbol{\tau}$, se utiliza la Eq. (17) para determinar el torsor $\widehat{\boldsymbol{w}}=\left(\boldsymbol{f}^{\top}, \boldsymbol{m}_{O}^{\top}\right)^{\top}$ que la bola efectúa sobre la plataforma.

3. Se aplica la Eq. (18) para obtener el vector de posición absoluto de $R, \boldsymbol{r}_{R}$.

4. Finalmente, se utiliza la Eq. (19) para obtener $\boldsymbol{r}_{R}^{\mathcal{P}}$ y por tanto sus componentes $e_{x} \mathrm{y} e_{y}$.

\subsection{CORRECCIÓN DE LA POSICIÓN}

La rama superior de la Fig. 3 describe, de izquierda a derecha, el flujo de cálculos involucrados en la corrección del error. Los errores $e_{x} \mathrm{y} e_{y}$ se introducen en dos controladores PID de tiempo discreto, dando como resultado dos incrementos de posición $\delta x$ y $\delta y$ en ambos ejes. El controlador que corrige $e_{x}$ implementa la siguiente ley de control

$$
\delta x=k_{p} e_{n}+k_{d}\left(e_{n}-e_{n-1}\right)+k_{i} \sum_{i=-\infty}^{i=n} e_{i}
$$

donde $e_{n}$ es el valor de $e_{x}$ en el $n$-ésimo instante de tiempo, y $k_{p}, k_{i}$, y $k_{d}$ son las constantes proporcional, integral, y derivativa, respectivamente. El controlador para $e_{y}$ implementa una ley análoga.

A partir de estos incrementos, se asignará a la plataforma un eje y un ángulo de giro. El primer paso es obtener el eje de rotación.
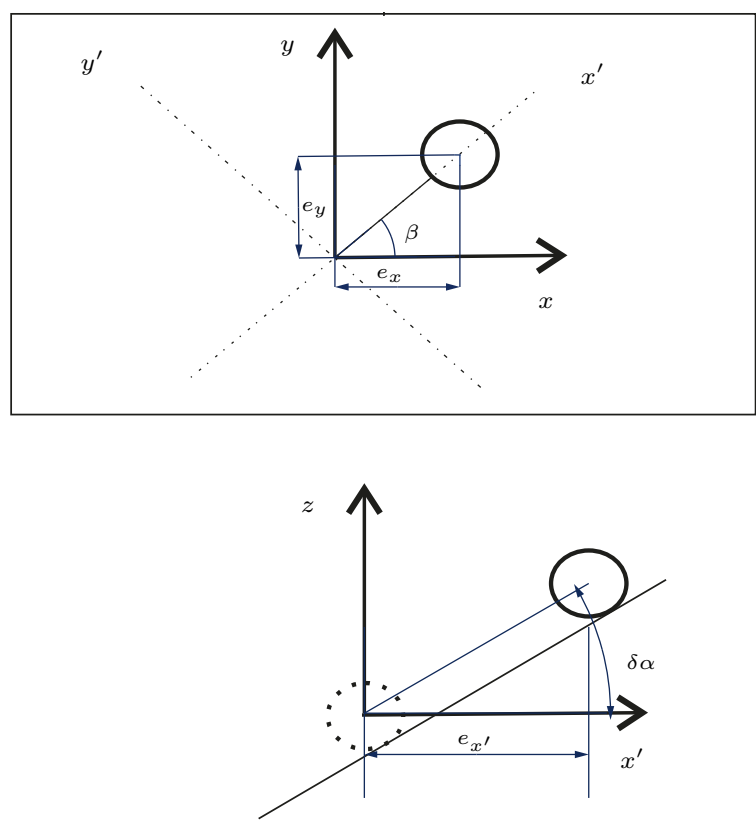

Figura 4: Eje y ángulo de rotación

$$
\begin{gathered}
\beta=t g^{-1} \frac{\delta y}{\delta x} \\
\boldsymbol{u}=(-\sin \beta) \hat{x}+(\cos \beta) \hat{y}
\end{gathered}
$$

Donde $\boldsymbol{u}$ es un vector director del eje de rotación. Es necesario también asignar un ángulo de rotación, que se hará en base a la norma del vector definido por los errores en ambos ejes. Para ello, se utilizará una función lineal con un valor de saturación que asigne un ángulo máximo para un valor de la norma mayor a una distancia $D$. Teniendo en cuenta que la norma del error viene dada por $\delta r=\sqrt{\delta x^{2}+\delta y^{2}}$

$$
\delta \alpha= \begin{cases}\alpha_{\max } \frac{\delta r}{D} & \delta r \leq D \\ \alpha_{\max } & \delta r>D\end{cases}
$$

Con la dirección del eje instantáneo de rotación y 
el incremento de giro, ya es posible definir el torsor $\delta \widehat{\boldsymbol{D}}$. Teniendo en cuenta que a la plataforma se le infringe una rotación pura alrededor de su eje, $\delta \widehat{\boldsymbol{D}}_{t}$ será nulo en todo momento.

$$
\delta \widehat{D}=\delta \alpha\left[\begin{array}{c}
\boldsymbol{O O}^{\prime} \times \boldsymbol{u} \\
\boldsymbol{u}
\end{array}\right]
$$

Siendo el vector $\boldsymbol{O} \boldsymbol{O}^{\prime}$ la relación entre el sistema de referencia de la base fija con el sistema de referencia utilizado para medir el error. $\delta \widehat{\boldsymbol{D}}$ será la entrada a la matriz jacobiana descrita en la ecuación (7), dando como resultado el incremento de los ángulos en los seis servos necesario para corregir la posición.

\section{IMPLEMENTACIÓN}

Para implementar los actuadores del robot se han utilizado seis servomotores Dynamixel AX12. Para los enlaces que conectan las articulaciones esféricas se han utilizado barras de nylon de $62 \mathrm{~mm}$ de diámetro. Estas barras sólo transmiten fuerzas a lo largo de sus ejes por estar ancladas por sus extremos a través de rótulas esféricas. Sin embargo, cuando se realizan pruebas de funcionamiento con robots paralelos, no es raro que por error se alcancen los límites articulares de sus juntas esféricas, transmitiendo pares de fueza a través de las barras que puedan dañar el propio robot. La utilización de barras flexibles se ha revelado de gran utilidad ya que permiten que las rótulas esféricas alcancen sus limites articulares sin que por ello el robot sufra daños.

La base y la plataforma del robot, ambas de forma hexagonal, se han materializado mediante una impresora 3D. En la base, los servomotores están colocados sobre los vértices del hexágono, de manera paralela a cada uno de los lados mayores. Las articulaciones se unen a cada uno de los actuadores a través de unos brazos que, en la posición de referencia, apuntan hacia el centro de cada lado mayor de la base hexagonal. Estos brazos también se han materializado con una impresora 3D. Las juntas esféricas son elementos estándar de bajo coste.

Sobre la plataforma móvil se ha fijado una plancha rectangular de $160 \times 120 \mathrm{~mm}$ sobre la que rodará una bola de de $20 \mathrm{~mm}$ de radio y 30 gramos de peso.

Para probar el sistema, y poder aislar el origen de los posibles problemas que puedan aparecer, la posición de la bola se obtiene también con una cámara USB PSP Eye, con una resolución de $640 \times 480$ píxeles, colocada en posición cenital a $50 \mathrm{~cm}$ del plano sobre el que rueda la bola, tal como se muestra en la Fig. 5. Utilizando el paquete OpenCV,

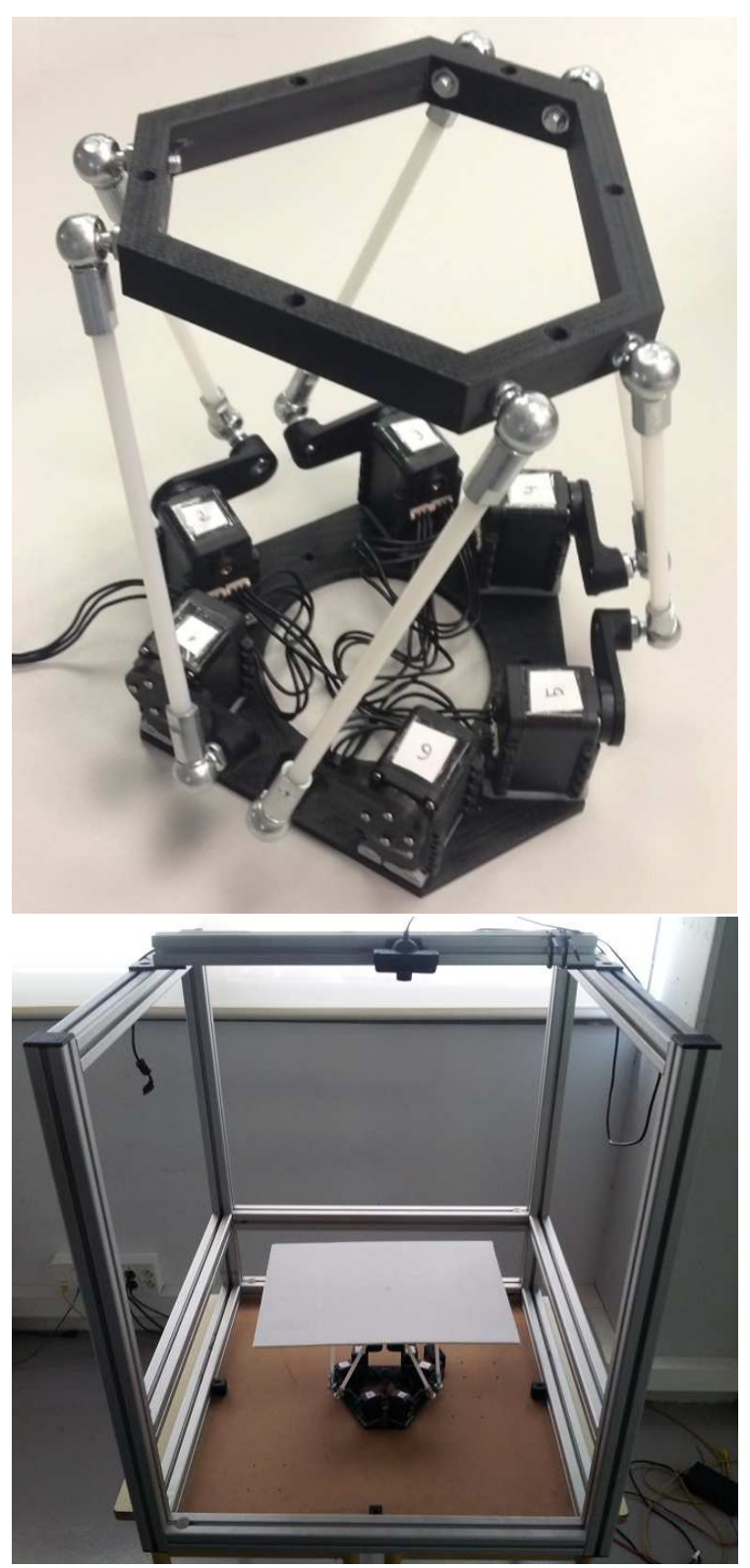

Figura 5: Robot paralelo construido, y su situación en el sistema experimental.

se obtiene la posición del centro de gravedad de la bola. Dado que esta posición se obtiene en coordenadas del plano de la imagen, es necesario una transformación que nos devuelva las cordenadas de la bola en el sistema de referencia del mundo utilizando la cinemática del robot descrita en la Sección 2.

Todo el software desarrollado para llevar a cabo este experimento ha sido escrito en lenguaje $\mathrm{C}++$, debido principalmente a su eficiencia y portabilidad. Cabe también mencionar que, debido a que los servomotores Dynamixel AX-12 poseen su propio protocolo de comunicación, se ha tenido que crear una librería de comunicaciones. 

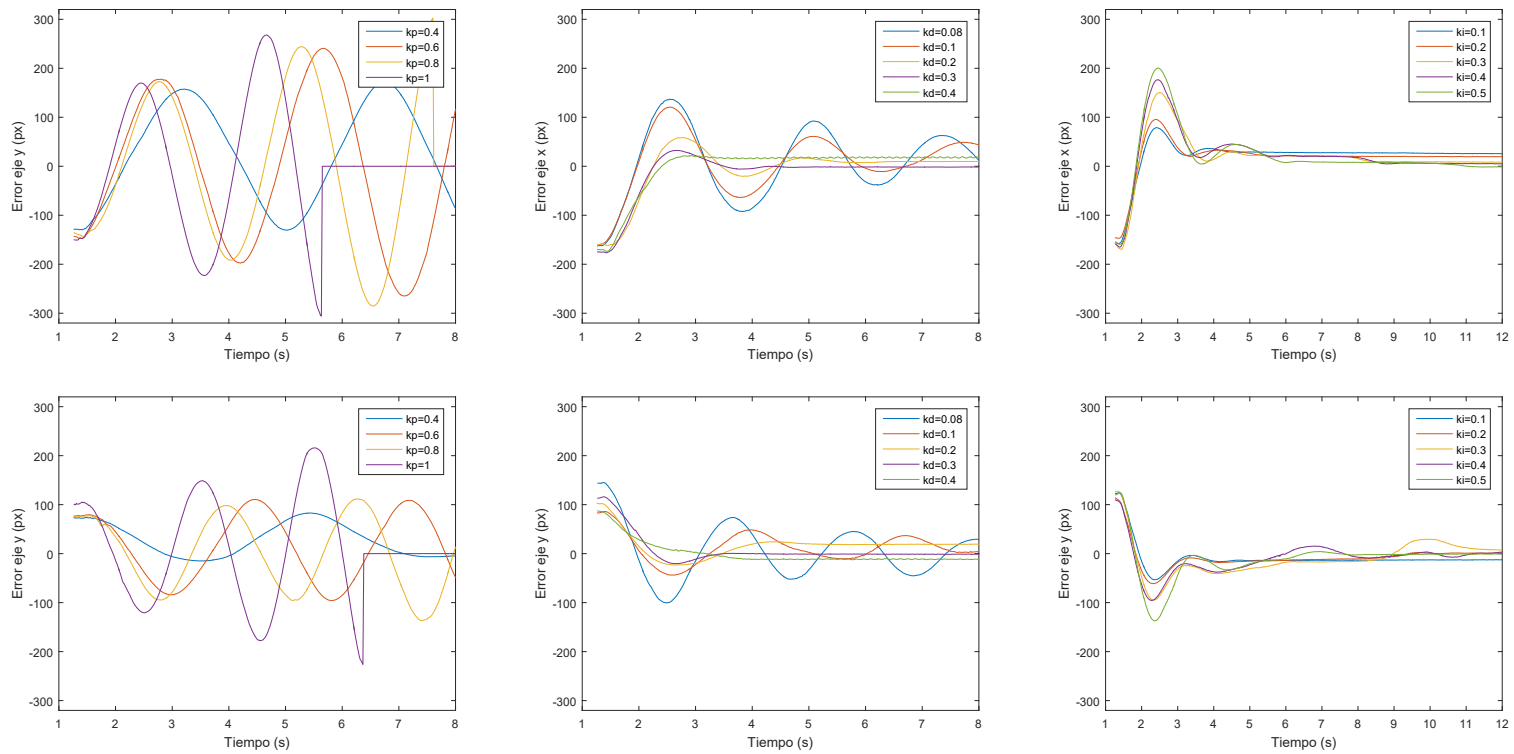

Figura 6: Influencia de $k_{p}, k_{d}$ y $k_{i}$ sobre la salida del sistema

En el momento en que fue redactado este trabajo, se encontraba operativo el bucle de control utilizando una cámara. Queda, por tanto, pendiente cerrar el bucle de control utilizando la información de fuerza y comparar los resultados obtenidos con los resultantes de utilizar el sistema de visión. A continuación, veremos, por tanto, cómo los parámetros del sistema de control influyen sobre las salidas del sistema utilizando únicamente el sistema de visión.

A medida que se aumenta el valor de $k_{p}$, llega un punto en que el sistema se hace inestable. Esta inestabilidad queda patente en la Fig. 6, donde para $k_{p}=1$ y aproximadamente en $t=5,6$ s la bola sale del campo de visión y la plataforma vuelve a la posición de reposo. A continuación, con la introducción de una ganancia diferencial, se tratará de corregir esta inestabilidad.

Eligiendo un valor de $k_{p}=0,8$ e introduciendo una ganancia diferencial, se comprueba como el tiempo de estabilización se reduce, a medida que $k_{d}$ aumenta. Sin embargo, a partir de cierto valor, pequeñas variaciones del error se traducen en picos en la salida del sistema, haciendo que la plataforma oscile con altas frecuencias alrededor de su punto de equilibrio. Un valor de $k_{d}=0,3$ nos sitúa en un comportamiento adecuado.

Debido al rozamiento seco entre la bola y la superficie obtenemos un error en régimen permanente que no puede ser compensado con un mero ajuste de la constante $k_{i}$, debido a la naturaleza no lineal del fenómeno. Por consiguiente, en la implementación actual se ha fijado $k_{i}=0$, ya que cualquier otro valor no aporta una mejora sustancial.
En una planta didáctica, una posible manera de limitar el error en régimen permanente sería elegir otros materiales que redujeran el rozamiento seco entre la plataforma y la bola, en cuyo caso tendría sentido buscar un valor óptimo de $k_{i}$.

\section{CONCLUSIONES}

En este artículo nos hemos centrado en el diseño, análisis e implementación de un robot paralelo 6RSS para la estabilización de la posición de una bola sobre un plano. Aunque el sistema es de bajo coste, proporciona una gran versatilidad en la actuación, y resuelve a la vez el problema del sensado de posición de la bola siguiendo las ideas presentadas en [8].

Se ha mostrado cómo la utilización de la teoría de torsores para formular el problema de la actuación y del sensado conduce a una formulación simple y unificada de ambos problemas.

La utilización del sistema propuesto tiene dos ventajas fundamentales. Por un lado, al disponer de una actuación que permite ejecutar torsores de rotación instantánea arbitrarios, abre la posibilidad a la utilización de estrategias de control mucho más sofisticadas a las que se han venido utilizando hasta el momento. Por otro lado, el disponer de la posición de la bola de forma prácticamente directa, sin la necesidad de procesar importante volúmenes de información como sucede cuando se utilizan imágenes, se eliminan retardos que degradan el comportamiento de cualquier estrategia de control que no los tenga en cuenta. 


\section{Referencias}

[1] S. Awtar, C. Bernard, N. Boklund, A. Master, D. Ueda, and K. Craig. Mechatronic design of a ball-on-plate balancing system. $\mathrm{Me}$ chatronics, 12(2):217-228, 2002.

[2] O. Bohigas, M. Manubens, and L. Ros. Singularities of non-redundant manipulators: A short account and a method for their computation in the planar case. Mechanism and Machine Theory, 68:1-17, 2013.

[3] O. Bohigas, M. Manubens, and L. Ros. Singularities of robot mechanisms: numerical computation and avoidance path planning, volume 41 of Mechanisms and Machine Science. Springer, 2016.

[4] O. Bohigas, D. Zlatanov, L. Ros, M. Manubens, and J. M. Porta. A general method for the numerical computation of manipulator singularity sets. IEEE Transactions on Robotics, 30(2):340-351, 2014.

[5] J. K. Davidson and K. J. Hunt. Robots and screw theory: applications of kinematics and statics to robotics. Oxford University Press, 2004.

[6] J. Duffy. Statics and kinematics with applications to robotics. Cambridge University Press, 1996.

[7] X. Fan, N. Zhang, and S. Teng. Trajectory planning and tracking of ball and plate system using hierarchical fuzzy control scheme. Fuzzy Sets and Systems, 144(2):297-312, 2004.

[8] R. Frigola, L. Ros, F. Roure, and F. Thomas. A wrench-sensitive touch pad based on a parallel structure. In 2008 IEEE International Conference on Robotics and Automation, pages 3449-3454, May 2008.

[9] C. Ham and M. Taufiq. Development of a ball and plate system. In ASEE Annual Conference and Exposition Proceedings. ASEE Conferences, 2015.

[10] J. Hauser, S. Sastry, and P. Kokotovic. Nonlinear control via approximate input-output linearization: the ball and beam example. IEEE Transactions on Automatic Control, 37(3):392-398, 1992.

[11] A. Kassem, H. Haddad, and C. Albitar. Comparison between different methods of control of ball and plate system with 6-DOF Stewart platform. IFAC-PapersOnLine, 48(11):4752,2015 .
[12] H. Liu and Y. Liang. Trajectory tracking sliding mode control of ball and plate system. In 2010 2nd International Asia Conference on Informatics in Control, Automation and Robotics (CAR 2010), volume 3, pages 142145. IEEE, 2010.

[13] M. Moarref, M. Saadat, and G. Vossoughi. Mechatronic design and position control of a novel ball and plate system. In 2008 16th $\mathrm{Me}$ diterranean Conference on Control and Automation, pages 1071-1076, 2008.

[14] H. Pottmann and J. Wallner. Computational Line Geometry. Springer Berlin Heidelberg, 2010.

[15] E. F. Sinaga, E. B. Manurung, V. A. Chee, and A. Djajadi. Building and controlling a ball and plate system. In V. V. Das, J. Stephen, and Y. Chaba, editors, Computer Networks and Information Technologies: 2nd International Conference on Advances in Communication, Network, and Computing (CNC 2011), pages 600-608. Springer Berlin Heidelberg, 2011.

[16] Y. Wang, M. Sun, Z. Wang, Z. Liu, and Z. Chen. A novel disturbance-observer based friction compensation scheme for ball and plate system. ISA Transactions, 53(2):671678, 2014. 